\title{
Optimal Symmetric Rendezvous Search on Three Locations
}

\author{
Richard Weber \\ Centre for Mathematical Sciences, Wilberforce Road, Cambridge CB2 0WB \\ email: rrw1@cam.ac.uk www.statslab.cam.ac.uk/ rrw1
}

In the symmetric rendezvous search game played on $n$ locations two players are initially placed at two distinct locations. The game is played in discrete steps, at each of which each player can either stay where he is or move to a different location. The players share no common labelling of the locations. We wish to find a strategy such that, if both players follow it independently, then the expected number of steps until they are in the same location is minimized. Informal versions of the rendezvous problem have received considerable attention in the popular press. The problem was proposed by Steve Alpern in 1976 and it has proved notoriously difficult to analyse. In this paper we prove a 20 year old conjecture that the following strategy is optimal for the game on three locations: in each block of two steps, stay where you, or search the other two locations in random order, doing these with probabilities $1 / 3$ and $2 / 3$ respectively. This is now the first nontrivial symmetric rendezvous search game to be fully solved.

Key words: rendezvous search; search games; semidefinite programming

MSC2000 Subject Classification: Primary: 90B40; Secondary: 49N75, 90C22, 91A12, 93A14

OR/MS subject classification: Primary: Games/group decisions, cooperative; Secondary: Analysis of algorithms; Programming, quadratic

1. Symmetric rendezvous search on three locations In the symmetric rendezvous search game played on $n$ locations two players are initially placed at two distinct locations. The game is played in discrete steps, and at each step each player can either stay where he is or move to another location. The players wish to meet as quickly as possible but must use the same strategy. Perhaps this strategy is described in a handbook of which they both have copies. An optimal strategy must involve randomizing moves, since if the players move deterministically they will simply 'chase one another's tails' and never meet. The players have no common labelling of the locations, so a given player must choose the probabilities with which he moves to each of the locations at step $k$ as a function only of where he has been at previous steps $0, \ldots, k-1$. One simple strategy would be for each player to move to each of the $n$ locations with equal probability at each step. Since under this strategy the probability of meeting at each step is $1 / n$ the expected number of steps required to meet is $n$. However, as we shortly see, this is not optimal. Can the reader think of something better before reading further?

Let $T$ denote the number of the step on which the players meet, and let $\omega$ denote the minimum achievable value of $E T$. We call $\omega$ the 'rendezvous value' of the game. A long-standing conjecture of Anderson and Weber (1990) 8 is that for symmetric rendezvous search on three locations the rendezvous value is $\omega=5 / 2$. This rendezvous value is achieved by a type of strategy which is now usually called the Anderson-Weber strategy (AW). It is motivated by the fact that if symmetry could be broken then it would be optimal for one player to remain stationary while the other player tours all locations (the 'wait-for-mommy' strategy). For rendezvous search on $n$ locations the AW strategy specifies that in blocks of $n-1$ consecutive steps the players should randomize between either staying at their current location, or touring the other $n-1$ locations in random order, doing these with some probabilities $p$ and $1-p$ respectively. On three locations this means that in each successive block of two steps, each player should, independently of the other, either stay at his initial location or tour the other two locations in random order, doing these with respective probabilities $1 / 3$ and $2 / 3$. The expected meeting time $E T$ for the AW strategy is $5 / 2$, so this is an upper bound on the rendezvous value, $\omega \leq 5 / 2$.

Rendezvous search problems have a long history. One finds an early version in the 'Quo Vadis' problem of Mosteller (1965) [14, and recently in 'Aisle Miles', O'Hare (2006) [12. In 2007 a letter writer to the Guardian newspaper queried, "I lost my wife in the crowd at Glastonbury (a music festival). What is the best strategy for finding her?" A reader replied, "Start talking to an attractive woman. Your wife will reappear almost immediately."

The first formal definition of the symmetric rendezvous search game on $n$ locations is due to Steve Alpern who stated it as a 'telephone coordination problem' in a seminar in 1976 [1] (see also [2] and [3]): Imagine that in each of two rooms, there are $n$ telephones randomly strewn about. They are connected in a pairwise fashion by $n$ wires. At discrete times $t=0,1, \ldots$, players in each room pick up a phone and 
say 'hello'. They wish to minimize the time $t$ when they first pick up paired phones and can communicate. Similarly, Alpern has also posed the 'Mozart Café Problem' (2010) [4]: Two friends agree to meet for lunch at the Mozart Café in Vienna on the first of January, 2000. However on arriving at Vienna airport, they are told there are three (or n) cafés with that name, no two close enough to visit in the same day. So each day each can go to one of them, hoping to find his friend there.

[Note that in the above two problems the players make an initial choice, which might cause them to meet. So the rendezvous value differs from that in which the players are intially placed at distinct locations. For example, if the AW strategy is used in the Mozart Café problem, with $n=3$ then the expected time to rendezvous is $1+(1 / 3) 0+(2 / 3)(5 / 2)=8 / 3$.]

While such idealized problems are remote from any real-life search-and-rescue problem, their study can teach us about issues in coordination, distributed learning, and decentralized control that are important in application areas, such as shared wireless communication (c.f. [13, [17]).

Anderson and Weber, proved (quite easily) that the AW strategy, with $p=1 / 2$, is optimal for the symmetric rendezvous search game on two locations. This is the same as 'search at random'. This fact was also shown in the same year by Crawford and Haller (1990) 9] for what they called a 'coordination game', in which after each step the two occupied locations are revealed. When $n=2$ this revelation is automatic and so the problems are identical. The coordination game when $n=3$ ends in one step, by both players moving to the location at which neither is presently located. When $n>3$ it is solved by playing the $n=2$ game on the two initially occupied locations. Anderson and Weber conjectured that the AW strategy should be optimal for the symetric rendezvous game on three locations. Indeed, in [8] they presented what they thought to be a proof of this, but it was later found to have an unrepairable error. Subsequently, there have many attempts to prove the optimality of AW for three locations, and to find what might be optimal for $n$ locations, $n>2$. It has been shown, for example, that AW is optimal for three locations within restricted classes of Markovian strategies, such as those that must repeat in successive blocks of $k$ steps, where $k$ is small. See Alpern and Pikounis (2000) 7] (for optimality of AW amongst 2-Markovian strategies for rendezvous on three locations), and Fan (2009) [10] (for optimality of AW amongst 4-Markovian strategies for rendezvous on three locations, and amongst 3-Markovian strategies for rendezvous on four locations).

The principal result in this paper is Theorem 2.1, in which we establish that AW is optimal for the symmetric rendezvous search game on three locations. This becomes the first nontrivial game of its type to be fully solved. We hope readers will enjoy our analysis of this game. The proof of Theorem 2.1 draws on multiple tools in the kit of mathematics for operations research: probability, game theory, linear algebra, linear programming and semidefinite programming. In particular, this paper provides another example of the way that semidefinite programming can be used to obtain lower bounds for $\mathcal{N} \mathcal{P}$-hard problems.

In Section 3 we discuss the thinking that led to discovery of this proof. Section 4 discusses a different problem which can be solved by the same method. This is a problem due to John Howard and is about minimizing the expected time until two players' rendezvous on two locations when they are sure to overlook one another at the first time they are in the same location. Section 5 discusses some generalizations and intriguing open problems.

2. Optimality of the Anderson-Weber strategy Recall that we have defined $T$ as the step on which the two players meet. Let us begin by noting that $E T=\sum_{i=0}^{\infty} P(T>i)$. It would certainly be helpful if AW were to minimize individually every term in this sum. But, contrary to what we had conjectured for many years, this is not true. In particular, the AW strategy produces $P(T>4)=1 / 9$. However, one can find a strategy such that $P(T>4)=1 / 10$. This is somewhat of a surprise and it shows that $E T=\sum_{i=0}^{\infty} P(T>i)$ cannot be minimized simply by minimizing each term of the sum simultaneously.

With Junjie Fan, we gained greater computational experience of the problem by solving semidefinite programming problems that provide lower bounds. Such research would not have been computationally feasible when the problem was first studied in the 1980s. Our solutions of semidefinite programs led us to conjecture that AW minimizes $E\left[\min [T, k+1]=\sum_{i=0}^{k} P(T>i)\right.$. This is the expected rendezvous time in a problem in which if the players have not met after $k$ th steps, they are put together at time 
$k+1$. We denote the infimal value of this quantity by $\omega_{k}$. We show that AW achieves $\omega_{k}$ for all $k$, i.e., that it minimizes the truncated sum $\sum_{i=0}^{k} P(T>i)$ for all $k$. This is what we now show: $\left\{\omega_{k}\right\}_{k=0}^{\infty}=\left\{1, \frac{5}{3}, 2, \frac{20}{9}, \frac{7}{3}, \frac{65}{27}, \ldots\right\}$ with $\omega_{k} \rightarrow 5 / 2$.

ThEOREM 2.1 The Anderson-Weber strategy is optimal for the symmetric rendezvous search game on three locations, minimizing $E[\min \{T, k+1\}]$ to $\omega_{k}$ for all $k=1,2, \ldots$, where

$$
\omega_{k}= \begin{cases}\frac{5}{2}-\frac{5}{2} 3^{-\frac{k+1}{2}}, & \text { when } k \text { is odd, } \\ \frac{5}{2}-\frac{3}{2} 3^{-\frac{k}{2}}, & \text { when } k \text { is even. }\end{cases}
$$

Consequently, the minimal achievable value of ET is $\omega=5 / 2$.

Before proceeding to the guts of the proof, we prepare with some preliminary ideas and notation. We begin by noting that the AW strategy is not uniquely optimal. There are infinitely many variations that are just as good. For example, it would be just as good if, in each block of two steps, a player were to either spend both steps at the location where he began at time 0 , or visit in random order the other two locations, again doing these with probabilities $1 / 3$ and $2 / 3$ respectively. We have simply replaced in the definition of AW the role of 'current location' with that of 'initial location'. In the proof that follows it is the 'initial location' form that we show is optimal, i.e. during any block of two steps in which a player stays in the same location he chooses this location to be that where he began at time 0 .

Suppose that the three locations are arranged around a circle and that the players have a common notion of clockwise. Such a common notion of clockwise might help. However, we shall prove that even if the players have a common notion of clockwise the AW strategy cannot be bettered, and this strategy makes no use of the clockwise information, the AW must also be optimal when the players do not have a common notion of clockwise.

Throughout most of what follows a subscript $k$ on a vector means that its length is $3^{k}$. A subscript $k$ on a matrix means that it is $3^{k} \times 3^{k}$. Let us define

$$
B_{k}=B_{1} \otimes B_{k-1}, \quad \text { where } B_{1}=\left(\begin{array}{lll}
1 & 1 & 0 \\
0 & 1 & 1 \\
1 & 0 & 1
\end{array}\right) .
$$

Here ' $\otimes$ ' denotes the Kronecker product. It is convenient to label the rows and columns of $B_{1}$ as $0,1,2$ (rather than 1,2,3). Suppose player II is initially placed one position clockwise of player I. Then $B_{1}(i, j$ ) is an indicator for the event that the players do not meet when at the first step player I moves $i$ positions clockwise from his initial location, and player II moves $j$ positions clockwise from his initial location. $B^{\top}$ contains the indicators for the same event, but when player II starts two positions clockwise of player I. Let $1_{k}$ denote the length $3^{k}$ column vector of $1 \mathrm{~s}$. Since the starting position of player II is randomly chosen, the problem of minimizing the probability of not having met after the first step is that of minimizing

$$
p^{\top}\left(\frac{1}{2}\left(B_{1}+B_{1}^{\top}\right)\right) p
$$

over $p \in \Delta_{1}$, where $\Delta_{k}$ is the standard simplex of probability vectors: $\Delta_{k}=\left\{p: p \in \mathbb{R}^{3 k}, p \geq\right.$ 0 and $\left.1_{k}^{\top} p=1\right\}$. Similarly, the 9 rows and 9 columns of $B_{2}$ can be labelled as $0, \ldots, 8$ (base 10 ), or $00,01,02,10,11,12,20,21,22$ (base 3 ). The base 3 labelling is helpful, for we may understand $B_{2}\left(i_{1} i_{2}, j_{1} j_{2}\right)$ as an indicator for the event that the players do not meet when at his first and second steps player I moves to locations that are respectively $i_{1}$ and $i_{2}$ positions clockwise from his initial position, and player II moves to locations that are respectively $j_{1}$ and $j_{2}$ positions clockwise from his initial position. The problem of minimizing the probability that they have not met after $k$ steps is that of minimizing

$$
p^{\top}\left(\frac{1}{2}\left(B_{k}+B_{k}^{\top}\right)\right) p=p^{\top} B_{k} p .
$$

It is helpful to adopt the notation that a bar over a square matrix denotes the symmetric matrix that is the average of that matrix and its transpose. That is, $\bar{A}=\frac{1}{2}\left(A+A^{\top}\right)$. Let $J_{k}$ be the $3^{k} \times 3^{k}$ matrix of all 1s. Let us try to choose $p$ to minimize

$$
E[\min \{T, k+1\}]=\sum_{i=0}^{k} P(T>i)=p^{\top} M_{k} p
$$


where

$$
\begin{aligned}
& M_{1}=J_{1}+B_{1} \\
& M_{k}=J_{k}+B_{1} \otimes M_{k-1}=J_{k}+B_{1} \otimes J_{k-1}+\cdots+B_{k-1} \otimes J_{1}+B_{k} .
\end{aligned}
$$

So

$$
\omega_{k}=\min _{p \in \Delta_{k}}\left\{p^{\top} M_{k} p\right\}=\min _{p \in \Delta_{k}}\left\{p^{\top} \bar{M}_{k} p\right\} .
$$

It is difficult to find the minimizing $p$, because $\bar{M}_{k}$ is not positive definite for $k \geq 2$. The quadratic form $p^{\top} M_{k} p$ has many local minima that are not global minima. For example, the strategy which randomizes equally over the three locations at each step, taking $p=1_{k} / 3^{k}=(1,1 \ldots, 1)^{\top} / 3^{k}$, is a local minimum of this quadratic form, but not the global minimum.

Let us consider in more detail the case $k=2$. To show that $\omega_{2}=2$ we must show this is the minimum of $p^{\top} \bar{M}_{2} p$. However, the eigenvalues of $\bar{M}_{2}$ are $\left\{19, \frac{5}{2}, \frac{5}{2}, 1,1,1,1,-\frac{1}{2},-\frac{1}{2}\right\}$, so, as remarked above, this matrix is not positive semidefinite. In general, the minimization over $x$ of a quadratic form such as $x^{\top} A x$ is $\mathcal{N} \mathcal{P}$-hard if $\bar{A}$ is not positive semidefinite. An alternative approach might be to try to show that $\bar{M}_{2}-2 J_{2}$ is a copositive matrix. For general $k$, this requires showing that $x^{\top}\left(\bar{M}_{2}-\omega_{k} J_{k}\right) x \geq 0$ for all $x \geq 0$, where $\left\{\omega_{k}\right\}_{k=1}^{\infty}=\left\{\frac{5}{3}, 2, \frac{20}{9}, \frac{7}{3}, \frac{65}{27}, \ldots\right\}$ are the values obtained by the Anderson-Weber strategy. However, to check copositivity numerically is also $\mathcal{N} \mathcal{P}$-hard.

One line of attack is a numerical approach based on semidefinite programming which finds a lower bound for $\omega_{k}$. Consider that

$$
\omega_{k}=\min _{p \in \Delta_{k}}\left\{p^{\top} M_{k} p\right\} \geq \min _{p \in \Delta_{k}}\left\{p^{\top} X_{k} p\right\}
$$

for any matrix $X_{k}$ such that $X_{k} \geq 0$ and $M_{k} \geq X_{k}$ (since these imply $p^{\top} M_{k} p \geq p^{\top} X_{k} p$ for all $p \geq 0$ ). Suppose we further restrict $X_{k}$ to be such that $\bar{X}_{k}$ is a positive semidefinite matrix (written $\bar{X}_{k} \succeq 0$ ), and also require that $p^{\top} \bar{X}_{k} p$ is minimized by the specific strategy $p^{\top}=1_{k} / 3^{k}$ (the random strategy). That is, we have the Kuhn-Tucker conditions $\bar{X}_{k}\left(1_{k} / 3^{k}\right)=\omega 1_{k}$, for some $\omega$. This poses the problem of finding a greatest lower bound:

$$
\underset{\omega, X_{k}}{\operatorname{maximize}} \omega: X_{k} \geq 0, M_{k} \geq X_{k}, \bar{X}_{k} \succeq 0, \bar{X}_{k} 1_{k}=\left(3^{k} \omega\right) 1_{k}
$$

or equivalently

$$
\underset{X_{k}}{\operatorname{maximize}} 3^{-2 k} \operatorname{trace}\left(J_{k} X_{k}\right): X_{k} \geq 0, M_{k} \geq X_{k}, \bar{X}_{k} \succeq 0, \bar{X}_{k} 1_{k}=3^{-k} \operatorname{trace}\left(J_{k} X_{k}\right) 1_{k}
$$

The above is a semidefinite programming problem and it can be solved numerically. We have done this with MATLAB and sedumi [16]. For $k=2,3,4,5$, we find that the greatest lower bound is indeed equal to the conjectured value of $\omega_{k}$.

The key idea towards a proof for $k>5$ is to replace all the above numerical work by algebra. Thus our task is to exhibit a matrix $X_{k}$ such that $M_{k} \geq X_{k} \geq 0, \bar{X}_{k}$ is positive semidefinite, and $p^{\top} X_{k} p$ is minimized over $p \in \Delta_{k}$ to $\omega_{k}$, by $p^{\top}=1_{k} / 3^{k}$.

For example, for $k=3$ we may take

$$
M_{2}=\left(\begin{array}{lllllllll}
3 & 3 & 2 & 3 & 3 & 2 & 1 & 1 & 1 \\
2 & 3 & 3 & 2 & 3 & 3 & 1 & 1 & 1 \\
3 & 2 & 3 & 3 & 2 & 3 & 1 & 1 & 1 \\
1 & 1 & 1 & 3 & 3 & 2 & 3 & 3 & 2 \\
1 & 1 & 1 & 2 & 3 & 3 & 2 & 3 & 3 \\
1 & 1 & 1 & 3 & 2 & 3 & 3 & 2 & 3 \\
3 & 3 & 2 & 1 & 1 & 1 & 3 & 3 & 2 \\
2 & 3 & 3 & 1 & 1 & 1 & 2 & 3 & 3 \\
3 & 2 & 3 & 1 & 1 & 1 & 3 & 2 & 3
\end{array}\right) \geq X_{2}=\left(\begin{array}{lllllllll}
3 & 3 & 2 & 3 & 3 & 2 & 1 & 1 & 0 \\
2 & 3 & 3 & 2 & 3 & 3 & 0 & 1 & 1 \\
3 & 2 & 3 & 3 & 2 & 3 & 1 & 0 & 1 \\
1 & 1 & 0 & 3 & 3 & 2 & 3 & 3 & 2 \\
0 & 1 & 1 & 2 & 3 & 3 & 2 & 3 & 3 \\
1 & 0 & 1 & 3 & 2 & 3 & 3 & 2 & 3 \\
3 & 3 & 2 & 1 & 1 & 0 & 3 & 3 & 2 \\
2 & 3 & 3 & 0 & 1 & 1 & 2 & 3 & 3 \\
3 & 2 & 3 & 1 & 0 & 1 & 3 & 2 & 3
\end{array}\right),
$$

where $\bar{X}_{2}$ is positive semidefinite, with eigenvalues $\left\{18,3,3, \frac{3}{2}, \frac{3}{2}, 0,0,0,0\right\}$. It is not hard to show that the minimum value of $p^{\top} \bar{X}_{2} p$ is 2 , which proves $\omega_{k}=2$. It is interesting that the minimum is achieved 
both by $p^{\top}=(1 / 9)(1,1,1,1,1,1,1,1,1)$ (the random strategy), and by $p^{\top}=(1 / 3)(1,0,0,0,0,1,0,1,0)$ (the AW strategy). We are now ready to present the proof of Theorem 2.1.

Proof of Theorem 2.1. In the above preliminaries we have shown that, with $M_{k}$ as defined by (3), the theorem is proved if for each $k$ we can find a matrix $X_{k}$ such that

(i) $M_{k} \geq X_{k} \geq 0$,

(ii) $\bar{X}_{k}$ is positive semidefinite, and

(iii) $p^{\top} X_{k} p$ is minimized over $p \in \Delta_{k}$ to $\omega_{k}$, by $p^{\top}=1_{k} / 3^{k}$.

Guided by our experience with extensive numerical experimentation, we make a guess that we may restrict our search for $X_{k}$ to matrices of the following special form. For $i=0, \ldots, 3^{k}-1$ we write $i_{\text {base } 3}=i_{1} \cdots i_{k}$ (always keeping $k$ digits, including leading 0 s when $i \leq 3^{k-1}-1$ ); so $i_{1}, \ldots, i_{k} \in\{0,1,2\}$. We define

$$
P_{i}=P_{i_{1} \cdots i_{k}}=P_{1}^{i_{1}} \otimes \cdots \otimes P_{1}^{i_{k}},
$$

where

$$
P_{1}=\left(\begin{array}{lll}
0 & 1 & 0 \\
0 & 0 & 1 \\
1 & 0 & 0
\end{array}\right) .
$$

Note that the subscript is now used for something other than the size of the matrix. It will always be easy for the reader to know the $k$ for which $P_{i}$ is $3^{k} \times 3^{k}$ by context. Observe that $M_{k}=\sum_{i} m_{k}(i) P_{i}$, where $m_{k}^{\top}=\left(m_{k}(0), \ldots, m_{k}\left(3^{k}-1\right)\right)=\left(M_{k}(0,0), \ldots, M_{k}\left(0,3^{k}-1\right)\right)$ denotes the top row of $M_{k}$. This motivates a search for an appropriate $X_{k}$ amongst those of the form

$$
X_{k}=\sum_{i=0}^{3^{k}-1} x_{k}(i) P_{i} .
$$

Let $x_{k}$ be the column vector $\left(x_{k}(0), \ldots, x_{k}\left(3^{k}-1\right)\right)^{\top}$. We claim that (i), (ii) and (iii) are equivalent, respectively, to conditions (i) ${ }^{\prime}$, (ii) ${ }^{\prime}$ and (iii) ${ }^{\prime}$, that we now present below.

(i) $)^{\prime} m_{k} \geq x_{k} \geq 0$.

The equivalence of (i) and (i) $)^{\prime}$ is trivial. In the example above, $X_{2}=\sum_{i} x_{2}(i) P_{i}$, where $x_{2}^{\top}=$ $(3,3,2,3,3,2,1,1,0)$, the first row of $X_{2}$.

To express (ii) in terms of $x_{k}$ requires something more subtle. We start with the important observation that the matrices $P_{0}, \ldots, P_{3^{k}-1}$ commute with one another and so have a common set of eigenvectors. Also, $P_{i}^{\top}=P_{i^{\prime}}$, where $i_{\text {base } 3}^{\prime}=i_{1}^{\prime} \cdots i_{k}^{\prime}$ is obtained from $i_{\text {base } 3}=i_{1} \cdots i_{k}$ by letting $i_{j}^{\prime}$ be $0,2,1$ as $i_{j}$ is $0,1,2$, respectively.

Let the columns of the matrices $U_{k}$ and $W_{k}$ contain the common eigenvectors of the $P_{i}$. Since $\bar{M}_{k}$ is a linear combination of the $P_{i}$ these are also the eigenvectors of $\bar{M}_{k}$. The columns of $W_{k}$ are eigenvectors with eigenvalues of 0 .

The eigenvalues of $\bar{X}_{k}$ are the same as the real parts of the eigenvalues of $X_{k}$. The eigenvectors and eigenvalues of $X_{k}$ can be computed as follows. Let $\omega$ be the cube root of 1 that is $\omega=-\frac{1}{2}+i \frac{1}{2} \sqrt{3}$. Then

$$
V_{k}=V_{1} \otimes V_{k-1}, \quad \text { where } V_{1}=\left(\begin{array}{ccc}
1 & 1 & 1 \\
1 & \omega & \omega^{2} \\
1 & \omega^{2} & \omega
\end{array}\right) .
$$

We write $V_{k}=U_{k}+i W_{k}$, and make use of the facts that $U_{k}=U_{1} \otimes U_{k-1}-W_{1} \otimes W_{k-1}$ and $W_{k}=$ $U_{1} \otimes W_{k-1}+W_{1} \otimes U_{k-1}$. It is easily checked that the eigenvectors of $P_{i}$ are the columns (and rows) of the symmetric matrix $V_{k}$ and that the first row of $V_{k}$ is $(1,1, \ldots, 1)$. The eigenvalues are also supplied in $V_{k}$, because if $V_{k}(j)$ denotes the $j$ th column of $V_{k}$ (an eigenvector), we have $P_{i} V_{k}(j)=V_{k}(i, j) V_{k}(j)$. Thus the corresponding eigenvalue is $V_{k}(i, j)$. Since $X_{k}$ is a sum of the $P_{i}$, we also have $X_{k} V_{k}(j)=$ $\sum_{i} x_{k}(i) V_{k}(i, j) V_{k}(j)$, so the eigenvalue is $\sum_{i} x_{k}(i) V_{k}(i, j)$, or $\sum_{i} V_{k}(j, i) x_{k}(i)$ since $V_{k}$ is symmetric. Thus the real parts of the eigenvalues of $X_{k}$ are the elements of the vector $U_{k} x_{k}$. This is nonnegative if and only if the symmetric matrix $\bar{X}_{k}$ is positive semidefinite. Thus the condition $\bar{X}_{k} \succeq 0$ is equivalent to

(ii) $U_{k} x_{k} \geq 0$. 
Finally, we turn to (iii). It is equivalent to

(iii) ${ }^{\prime} x_{k}^{\top}\left(1_{k} / 3^{k}\right)=\omega_{k}$.

This is because $p^{\top} X_{k} p$ is minimized to $\omega_{k}$, by $p^{\top}=1_{k} / 3^{k}$ if we have the Kuhn-Tucker condition $X_{k} 1_{k} / 3^{k}=\omega_{k} 1_{k}$. Recall that $1_{k}$ denotes the length $3^{k}$ column vector of $1 \mathrm{~s}$. Since $P_{i} 1_{k}=1$, the Kuhn-Tucker condition is $x_{k}^{\top}\left(1_{k} / 3^{k}\right)=\omega_{k}$. That is, the sum of the components of $x_{k}$ should be $3^{k} \omega_{k}$.

Having determined that the theorem will be proved once we find $x_{k}$ satisfying (i) ${ }^{\prime}$, (ii)' , and (iii)' , we are now ready to state an $x_{k}$ that will prove the theorem. In Section 3 we will say something about how that following recursion was discovered.

$$
\begin{aligned}
& x_{1}=(2,2,1)^{\top} \\
& x_{2}=(3,3,2,3,3,2,1,1,0)^{\top} \\
& x_{k}=1_{k}+(1,0,0)^{\top} \otimes x_{k-1}+(0,1,0)^{\top} \otimes\left(\alpha_{k}, \alpha_{k}, 2,2, \alpha_{k}, 2,1,1,1\right)^{\top} \otimes 1_{k-3}, \quad k \geq 3 .
\end{aligned}
$$

The parameter $\alpha_{k}$ is chosen so that (iii) ${ }^{\prime}$ is satisfied, i.e. $x_{k}^{\top}\left(1_{k} / 3^{k}\right)=\omega_{k}$, for $\omega_{k}$ specified by (1). Since the sum of the components of $x_{k}$ is

$$
x_{k}^{\top} 1_{k}=3^{k}+x_{k-1}^{\top} 1_{k-1}+3^{k-2}\left(3+\alpha_{k}\right)
$$

we find that we need the $\alpha_{k}$ to be:

$$
\alpha_{k}= \begin{cases}3-\frac{1}{3^{(k-3) / 2}}, & \text { when } k \text { is odd, } \\ 3-\frac{2}{3^{(k-2) / 2}}, & \text { when } k \text { is even. }\end{cases}
$$

So

$$
\left\{\alpha_{3}, \alpha_{4}, \ldots, \alpha_{11}, \ldots\right\}=\left\{2, \frac{7}{3}, \frac{8}{3}, \frac{25}{9}, \frac{26}{9}, \frac{79}{27}, \frac{80}{27}, \frac{241}{81}, \frac{242}{81}, \ldots\right\} .
$$

Alternatively, the values of $3-\alpha_{k}$ are $1, \frac{2}{3}, \frac{1}{3}, \frac{2}{9}, \frac{1}{9}, \frac{2}{27}, \ldots$ For example, with $\alpha_{3}=2$ we have

$$
\begin{gathered}
m_{3}=(4,4,3,4,4,3,2,2,2,4,4,3,4,4,3,2,2,2,1,1,1,1,1,1,1,1,1)^{\top}, \\
x_{3}=(4,4,3,4,4,3,2,2,1,3,3,3,3,3,3,2,2,2,1,1,1,1,1,1,1,1,1)^{\top} .
\end{gathered}
$$

Note that $\alpha_{k}$ increases monotonically in $k$, from 2 towards 3 . As $k \rightarrow \infty$ we find $\alpha_{k} \rightarrow 3$ and $x_{k}^{\top}\left(1_{k} / 3^{k}\right) \rightarrow$ 5/2. By construction we have now ensured (iii)' and (iii). It remains to prove that with $\alpha_{k}$ defined in (7) we also have (i)' and (ii)'. These are equivalent to (i) $M_{k} \geq X_{k} \geq 0$ and (ii) $X_{k} \succeq 0$, which are sufficient for the theorem to be true.

Checking $(i)^{\prime}$. To prove $m_{k} \geq x_{k}$ is easy; we use induction. The base of the induction is $m_{2}=(3,3,2,3,3,2,1,1,1)^{\top} \geq x_{2}=(3,3,2,3,3,2,1,1,0)^{\top}$. Assuming $m_{k-1} \geq x_{k-1}$, we then have

$$
\begin{aligned}
m_{k}= & 1_{k}+(1,1,0)^{\top} \otimes m_{k-1} \\
\geq & 1_{k}+(1,0,0)^{\top} \otimes m_{k-1} \\
& +(0,1,0)^{\top} \otimes\left(1_{k-1}+(1,1,0)^{\top} \otimes 1_{k-2}+(1,1,0,1,1,0,0,0,0)^{\top} \otimes 1_{k-3}\right) \\
= & 1_{k}+(1,0,0)^{\top} \otimes m_{k-1}+(0,1,0)^{\top} \otimes(3,3,2,3,3,2,1,1,1)^{\top} \otimes 1_{k-3} \\
\geq & 1_{k}+(1,0,0)^{\top} \otimes x_{k-1}+(0,1,0)^{\top} \otimes\left(\alpha_{k}, \alpha_{k}, 2,2, \alpha_{k}, 2,1,1,1\right)^{\top} \otimes 1_{k-3} \\
= & x_{k} .
\end{aligned}
$$

Checking (ii)'. To prove $U_{k} x_{k} \geq 0$ is much harder. Indeed, $U_{k} x_{k}$ is barely nonnegative, in the sense that as $k \rightarrow \infty, 5 / 9$ of its components are 0 , and $2 / 9$ of them are equal to $3 / 2$. Thus most of the eigenvalues of $\bar{X}_{k}$ are 0 . We do not need this fact, but it is interesting that $2 U_{k} x_{k}$ is a vector only of integers. The calculations throughout the remainder of proof are straightforward in principle. However, the reader may find that formula involving Kronecker and outer products require careful inspection if one is fully to understand. One way we have checked that the identities below are correct is by doing everything with numbers and verifying that the formula give the right answers for $k=2,3,4,5$.

Let $f_{k}$ be a column vector of length $3^{k}$ in which the first component is 1 and all other components are 0 . Using the facts that $U_{k}=U_{1} \otimes U_{k-1}-W_{1} \otimes W_{k-1}=U_{3} \otimes U_{k-3}-W_{3} \otimes W_{k-3}$ and $W_{k} 1_{k}=0$ and $U_{k} 1_{k}=3^{k} f_{k}$, we have

$$
U_{2} x_{2}=\left(18, \frac{3}{2}, \frac{3}{2}, 3,0,0,3,0,0\right)^{\top},
$$


and for $k \geq 3$,

$$
\begin{aligned}
U_{k} x_{k} & =3^{k} f_{k}+(1,1,1)^{\top} \otimes U_{k-1} x_{k-1} \\
& +\left(U_{3}\left((0,1,0)^{\top} \otimes\left(\alpha_{k}, \alpha_{k}, 2,2, \alpha_{k}, 2,1,1,1\right)^{\top}\right)\right) \otimes U_{k-3} 1_{k-3} \\
& =3^{k} f_{k}+(1,1,1)^{\top} \otimes U_{k-1} x_{k-1}+3^{k-3} r_{k} \otimes f_{k-3},
\end{aligned}
$$

where $r_{k}$ is

$$
\begin{aligned}
& r_{k}=U_{3}\left((0,1,0)^{\top} \otimes\left(\alpha_{k}, \alpha_{k}, 2,2, \alpha_{k}, 2,1,1,1\right)^{\top}\right) \\
& \begin{array}{r}
=\frac{3}{2}\left(6+2 \alpha_{k}, 0,0, \alpha_{k}-1,0, \alpha_{k}-2, \alpha_{k}-1, \alpha_{k}-2,0\right. \\
-3-\alpha_{k}, 2-\alpha_{k}, \alpha_{k}-2,-\alpha_{k}, 0,0,1,2-\alpha_{k}, 0, \\
\left.\quad-3-\alpha_{k}, \alpha_{k}-2,2-\alpha_{k}, 1,0,2-\alpha_{k},-\alpha_{k}, 0,0\right)^{\top} .
\end{array}
\end{aligned}
$$

Note that we make a small departure from our subscripting convention, since $r_{k}$ is not of length $3^{k}$, but of length 27 . We use the subscript $k$ to denote that $r_{k}$ is a function of $\alpha_{k}$.

Using (8)-(11) it easy to compute the values $U_{k} x_{k}$, for $k=2,3, \ldots$. Notice that there is no need to calculate the $3^{k} \times 3^{k}$ matrix $U_{k}$. Computing $U_{k} x_{k}$ as far as $k=15$, we find that for the values of $\alpha_{k}$ conjectured in (7) we do indeed always have $U_{k} x_{k} \geq 0$. This gives a lower bound on the rendezvous value of $w \geq \omega_{15}=16400 / 6561 \approx 2.49962$. It would not be hard to continue to even larger $k$ (although $U_{15} x_{15}$ is already a vector of length $\left.3^{15}=14,348,907\right)$. Clearly the method is working. It now remains to prove that $U_{k} x_{k} \geq 0$ for all $k$.

Consider the first third of $U_{k} x_{k}$. This is found from (6) and (9) to be

$$
3^{k} f_{k-1}+U_{k-1} x_{k-1}+3^{k-3} \frac{3}{2}\left(6+2 \alpha_{k}, 0,0, \alpha_{k}-1,0, \alpha_{k}-2, \alpha_{k}-1, \alpha_{k}-2,0\right)^{\top} \otimes f_{k-3} .
$$

Assuming $U_{k-1} x_{k-1} \geq 0$ as an inductive hypothesis, and using the fact that $\alpha_{k} \geq 2$, this vector is nonnegative. So this part of $U_{k} x_{k}$ is nonnegative.

As for the rest of $U_{k} x_{k}$ (the part that can be found from (6) and (10)-(11)), notice that $r_{k}$ is symmetric, in the sense that $S_{3} r_{k}=r_{k}$, where

$$
S_{1}=\left(\begin{array}{ccc}
1 & 0 & 0 \\
0 & 0 & 1 \\
0 & 1 & 0
\end{array}\right)
$$

and $S_{3}=S_{1} \otimes S_{1} \otimes S_{1}$. The matrix $S_{k}$ transposes 1s and 2s. Indeed $S_{k} P_{i}=P_{i}^{\top}$. Thus the proof is complete if we can show that just the middle third of $U_{k} x_{k}^{\top}$ is nonnegative. Assuming that $U_{k-1} x_{k-1} \geq 0$ and $\alpha_{k} \geq 2$, there are just 4 components of this middle third that depend on $\alpha_{k}$ and which might be negative. Let $I_{k}$ denote a $3^{k} \times 3^{k}$ identity matrix. This middle third is found from (6) and $(10)$ and is as follows, where we indicate in bold face terms that might be negative,

$$
\begin{aligned}
((0,1,0) \otimes & \left.I_{k-1}\right) U_{k} x_{k} \\
& =U_{k-1} x_{k-1}+\frac{3}{2} 3^{k-3}\left(-\mathbf{3}-\boldsymbol{\alpha}_{\boldsymbol{k}}, \mathbf{2}-\boldsymbol{\alpha}_{\boldsymbol{k}}, \alpha_{k}-2,-\boldsymbol{\alpha}_{\boldsymbol{k}}, 0,0,1, \mathbf{2}-\boldsymbol{\alpha}_{\boldsymbol{k}}, 0\right)^{\top} \otimes f_{k-3} .
\end{aligned}
$$

The four possibly negative components of the middle third are shown above in bold and are

$$
\begin{aligned}
t_{k 1} & =(0,1,0) \otimes(1,0,0,0,0,0,0,0,0) \otimes f_{k-3}^{\top} U_{k} x_{k} \\
& =\left(U_{k-1} x_{k-1}\right)_{1}+\frac{3}{2} 3^{k-3}\left(-3-\alpha_{k}\right) \\
t_{k 2} & =(0,1,0) \otimes(0,1,0,0,0,0,0,0,0) \otimes f_{k-3}^{\top} U_{k} x_{k} \\
& =\left(U_{k-1} x_{k-1}\right)_{3^{k-3}+1}+\frac{3}{2} 3^{k-3}\left(2-\alpha_{k}\right) \\
t_{k 3} & =(0,1,0) \otimes(0,0,0,1,0,0,0,0,0) \otimes f_{k-3}^{\top} U_{k} x_{k} \\
& =\left(U_{k-1} x_{k-1}\right)_{33^{k-3}+1}+\frac{3}{2} 3^{k-3}\left(-\alpha_{k}\right) \\
t_{k 4} & =(0,1,0) \otimes(0,0,0,0,0,0,0,1,0) \otimes f_{k-3}^{\top} U_{k} x_{k} \\
& =\left(U_{k-1} x_{k-1}\right)_{73^{k-3}+1}+\frac{3}{2} 3^{k-3}\left(2-\alpha_{k}\right)
\end{aligned}
$$


The remainder of the proof is devoted to proving that all these are nonnegative. Consider $t_{k 1}$. It is easy to work out a formula for $t_{k 1}$, since

$$
\begin{aligned}
\left(U_{k} x_{k}\right)_{1} & =f_{k}^{\top} U_{k} x_{k} \\
& =3^{k}+f_{k-1}^{\top} U_{k-1} x_{k-1}+3^{k-3} \frac{3}{2}\left(6+2 \alpha_{k}\right) \\
& =\left(U_{k-1} x_{k-1}\right)_{1}+43^{k-1}+3^{k-2} \alpha_{k}
\end{aligned}
$$

Thus

and

$$
\left(U_{k} x_{k}\right)_{1}=23^{k}+\sum_{i=3}^{k} 3^{i-2} \alpha_{i}
$$

This is nonnegative since $\alpha_{k} \leq 3$.

$$
t_{k 1}=\frac{1}{2} 3^{k}+\sum_{i=3}^{k-1} 3^{i-2} \alpha_{i}-\frac{1}{2} 3^{k-2} \alpha_{k}
$$

Amongst the remaining terms, we observe from numerical work that $t_{k 2} \geq t_{k 4} \geq t_{k 3}$. This suggests that $t_{k 3}$ is the least of the four terms, and it constrains the size of $\alpha_{k}$. Let us begin therefore by finding a formula for $t_{k 3}$. We have

$$
\begin{aligned}
t_{k 3} & =(0,1,0) \otimes(0,0,0,1,0,0,0,0,0) \otimes f_{k-3}^{\top} U_{k} x_{k} \\
& =(0,0,0,1,0,0,0,0,0) \otimes f_{k-3}^{\top} U_{k-1} x_{k-1}-3^{k-2} \frac{1}{2} \alpha_{k} \\
& =(0,1,0) \otimes f_{1}^{\top} \otimes f_{k-3}^{\top}\left(3^{k-1} f_{k-1}+(1,1,1)^{\top} \otimes U_{k-2} x_{k-2}+3^{k-4} r_{k-1} \otimes f_{k-4}\right)-3^{k-2} \frac{1}{2} \alpha_{k} \\
& \left.=(1,0,0,0,0,0,0,0,0) \otimes f_{k-4}^{\top} U_{k-2} x_{k-2}+3^{k-4}(0,1,0) \otimes f_{2}\right) r_{k-1}-3^{k-2} \frac{1}{2} \alpha_{k} \\
& =\left(U_{k-2} x_{k-2}\right)_{1}-3^{k-4} \frac{3}{2}\left(3+\alpha_{k-1}\right)-3^{k-2} \frac{1}{2} \alpha_{k} \\
& =\left(U_{k-2} x_{k-2}\right)_{1}-3^{k-3} \frac{1}{2}\left(3+\alpha_{k-1}\right)-3^{k-2} \frac{1}{2} \alpha_{k}
\end{aligned}
$$

This means that $t_{k 3}$ can be computed from the first component of $U_{k-2} x_{k-2}$, which we have already found in 16 . So

$$
\begin{aligned}
t_{k 3} & =23^{k-2}+\sum_{i=3}^{k-2} 3^{i-2} \alpha_{i}-3^{k-3} \frac{1}{2}\left(3+\alpha_{k-1}\right)-3^{k-2} \frac{1}{2} \alpha_{k} \\
& =\frac{1}{2} 3^{k-1}+\sum_{i=3}^{k-2} 3^{i-2} \alpha_{i}-\frac{1}{2} 3^{k-3} \alpha_{k-1}-\frac{1}{2} 3^{k-2} \alpha_{k} .
\end{aligned}
$$

We now put the $\alpha_{k}$ to the values specified in (7). It is easy to check with (7) and (18) that $t_{k 3}=0$ for all $k$.

It remains only to check that also $t_{k 2} \geq 0$ and $t_{k 4} \geq 0$. We have

$$
\begin{aligned}
t_{k 2}= & (0,1,0) \otimes(0,1,0,0,0,0,0,0,0) \otimes f_{k-3}^{\top} U_{k} x_{k} \\
= & (0,1,0,0,0,0,0,0,0) \otimes f_{k-3}^{\top} U_{k-1} x_{k-1}+3^{k-2}\left(1-\frac{1}{2} \alpha_{k}\right) \\
= & (1,0,0) \otimes(0,1,0) \otimes f_{k-3}^{\top}\left(3^{k-1} f_{k-1}+(1,1,1)^{\top} \otimes U_{k-2} x_{k-2}+3^{k-4} r_{k-1} \otimes f_{k-4}\right) \\
& +3^{k-2}\left(1-\frac{1}{2} \alpha_{k}\right) \\
= & (0,1,0) \otimes f_{k-3}^{\top} U_{k-2} x_{k-2}-3^{k-4} \frac{3}{2}\left(1-\alpha_{k-1}\right)+3^{k-2}\left(1-\frac{1}{2} \alpha_{k}\right) .
\end{aligned}
$$

We recognize $(0,1,0) \otimes f_{k-3}^{\top} U_{k-2} x_{k-2}$ to be the first component of the middle third of $U_{k-2} x_{k-2}$. The recurrence relation for this is

$$
\begin{aligned}
(0,1,0) \otimes f_{k-1}^{\top} U_{k} x_{k} & =(0,1,0) \otimes f_{k-1}^{\top}\left(3^{k} f_{k}+(1,1,1)^{\top} \otimes U_{k-1} x_{k-1}+3^{k-3} r_{k} \otimes f_{k-3}\right) \\
& =f_{k-1}^{\top} U_{k-1} x_{k-1}-3^{k-2} \frac{1}{2}\left(3+\alpha_{k}\right) .
\end{aligned}
$$

The right hand side can be computed from 16 . So we now have,

$$
\begin{aligned}
t_{k 2} & =23^{k-3}+\sum_{i=3}^{k-3} 3^{i-2} \alpha_{i}-3^{k-4} \frac{1}{2}\left(3+\alpha_{k-2}\right)-3^{k-3} \frac{1}{2}\left(1-\alpha_{k-1}\right)+3^{k-2}\left(1-\frac{1}{2} \alpha_{k}\right) \\
& =43^{k-3}+\sum_{i=3}^{k-3} 3^{i-2} \alpha_{i}-\frac{1}{2} 3^{k-4} \alpha_{k-2}+\frac{1}{2} 3^{k-3} \alpha_{k-1}-\frac{1}{2} 3^{k-2} \alpha_{k} .
\end{aligned}
$$


Finally, we establish a formula for $t_{k 4}$.

$$
\begin{aligned}
t_{k 4}= & (0,1,0) \otimes(0,0,0,0,0,0,0,1,0) \otimes f_{k-3}^{\top} U_{k} x_{k} \\
= & (0,0,0,0,0,0,0,1,0) \otimes f_{k-3}^{\top} U_{k-1} x_{k-1}+3^{k-2}\left(1-\frac{1}{2} \alpha_{k}\right) \\
= & (0,0,1) \otimes(0,1,0) \otimes f_{k-3}^{\top}\left(3^{k-1} f_{k-1}+(1,1,1)^{\top} \otimes U_{k-2} x_{k-2}+3^{k-4} r_{k-1} \otimes f_{k-4}\right) \\
& +3^{k-2}\left(1-\frac{1}{2} \alpha_{k}\right) \\
= & (0,1,0) \otimes f_{k-3}^{\top} U_{k-2} x_{k-2}+3^{k-4} \frac{3}{2}+3^{k-2}\left(1-\frac{1}{2} \alpha_{k}\right) \\
= & 53^{k-3}+\sum_{i=3}^{k-3} 3^{i-2} \alpha_{i}-\frac{1}{2} 3^{k-4} \alpha_{k-2}-\frac{1}{2} 3^{k-2} \alpha_{k} .
\end{aligned}
$$

Now we can check the truth of the fact that we observed empirically, that $t_{k 2} \geq t_{k 4} \geq t_{k 3}$. We find

$$
\begin{aligned}
& t_{k 2}-t_{k 4}=\frac{1}{2} 3^{k-3}\left(\alpha_{k-1}-2\right), \\
& t_{k 4}-t_{k 3}=\frac{1}{2} 3^{k-3}\left(1-\alpha_{k-2}+\alpha_{k-1}\right) .
\end{aligned}
$$

Since $\alpha_{k}$ is at least 2 and $\alpha_{k}$ is increasing in $k$, both of the above are nonnegative. So $t_{k 2}$ and $t_{k 4}$ are both at least as great as $t_{k 3}$, which we have already shown to be 0 . This establishes $U_{k} x_{k} \geq 0$ and so the proof is now complete.

3. On discovery of the proof A careful reader of the above proof will surely feel that (6) begs a question. Where did this recursion for $x_{k}$ come from? It seems to have been plucked out of the air. Let us restate it here for convenience. With $\alpha_{k}$ given by (7), the recursion of $(6)$ is

$$
x_{k}=1_{k}+(1,0,0)^{\top} \otimes x_{k-1}+(0,1,0)^{\top} \otimes\left(\alpha_{k}, \alpha_{k}, 2,2, \alpha_{k}, 2,1,1,1\right)^{\top} \otimes 1_{k-3} .
$$

It is interesting that there are many choices of $x_{2}$ that will work. We could have taken $x_{2}^{\top}=$ $(3,3,2,2,3,2,1,1,1)$ or $x_{2}^{\top}=(3,3,2,3,2,2,1,1,1)$. Let us briefly describe the steps and ideas in research that led to 22 .

As mentioned above, we began our investigations by computing lower bounds on $\omega_{k}$ by solving (5). These turn out to be achieved by the AW strategy and so are useful in proving the Fan-Weber conjecture (that AW minimizes $E[\min \{T, k+1\}])$ up to $k=5$. However, these lower bounds only produce numerical answers, with little guide as to a general form of solution. In fact, since one can only solve the SDPs up to the numerical accuracy of a SDP solver (which, like sedumi [16, uses interior point methods), such proofs are only approximate. For example, by this method one can only prove that $\omega_{5} \geq 2.40740740$, but not $\omega_{5}=65 / 27=2.4074074 \ldots$.

One would like to find rational solutions so the proofs can be exact. A major breakthrough was to realise that we could compute a common eigenvector set for $P_{1}, \ldots, P_{3^{k}-1}$ and write $M_{k}=\sum_{i} m_{k}(i) P_{i}$. We discovered this as we noticed and tried to explain the fact that the real parts of all the eigenvalues of $2 M_{k}$ are integers. (In fact, this follows from the so-called rational roots theorem, which states that if a polynomial $a_{n} x^{n}+a_{n-1} x^{n-1}+\cdots+a_{0}$ has integer coefficients, and $p / q$ is a rational root expressed in integers $p$ and $q$ that have no common divisor, then $p \mid a_{0}$ and $q \mid a_{n}$ (see [15]). So if $A$ is a $n \times n$ matrix of integers then $\operatorname{det}(x I-A)$ is a polynomial with integer coefficients and $a_{n}=1$, so all rational eigenvalues of $A$ must be integers.) This allowed us to recast (5) as the linear program

$$
\underset{x_{k}=\left(x_{k}(0), \ldots, x_{k}\left(3^{k}-1\right)\right)}{\operatorname{maximize}} \sum_{i=0}^{3^{k}-1} x_{k}(i): x_{k} \geq 0, x_{k} \leq m_{k}, U_{k} x_{k} \geq 0 .
$$

Now we can find exact proofs of the Fan-Weber conjecture as far as $k=8$, where $U_{8}$ is a matrix of size $6561 \times 6561$. These solutions were found using Mathematica and were in rational numbers, thus providing us with proofs that AW minimizes $E[\min \{T, k+1\}]$, up to $k=8$. This is better than we would do with semindefinite programming because the number of decision variables in the linear program (23) grows as $3^{k}$, whereas in the semidefinite program (5) grows as $3^{2 k}$.

It seems very difficult to find a general solution to (23) that holds for all $k$. The LP is highly degenerate with many optimal solutions. There are indeed 12 different extreme point solutions even when $k$ is only 2. No general pattern to the solution emerges as it is solved for progressively larger $k$. For $k=4$ there are 
many different $X_{4}$ that can be used to prove $\omega_{k}=7 / 3$. So we searched amongst the many such solutions, looking for ones with some pattern that might be generalized. This proved very difficult. We tried forcing lots of components of the solution vector to be integers, or identical, and looked for solutions in which the solution vector for $k-1$ was embedded within the solution vector for $k$. We looked at adding other constraints, and constructed some solutions by augmenting the objective function and choosing amongst possible solution by a minimizing a sum of squares penalty.

Another approach to the problem of minimizing $p^{\top} M_{k} p$ over $p \in \Delta_{k}$ is to make the identification $Y=p p^{\top}$. With this identification, $Y$ is positive semidefinite, $\operatorname{trace}\left(J_{k} Y\right)=1$, and $\operatorname{trace}\left(M_{k} Y\right)=$ $\operatorname{trace}\left(M_{k} p p^{\top}\right)=p^{\top} M_{k} p$. This motivates a semidefinite programming relaxation of our problem: minimize $\operatorname{trace}\left(M_{k} Y\right)$, subject to trace $\left(J_{k} Y\right)=1$ and $Y \succeq 0$. This can be recast as the linear program:

$$
\text { minimize } m_{k} y: y^{\top} U_{k} \geq 0,1_{k}^{\top} y=1, y \geq 0 .
$$

This is nearly the dual of 23 .

With (24) in mind, we imagined taking $y$ to the the AW strategy and worked at trying to guess a full basis in the columns of $U_{k}$ that is complementary slack to $y$ and from which one can then compute a solution to (23). We also explored a number of further linear programming formulations. All of this was helpful in building up intuition as to how a general solution might possibly be constructed.

Another major breakthrough was to choose to work with the constraint $x \leq m_{k}$ in which $m_{k}$ is the first row of the nonsymmetric matrix $M_{k}$, rather than to use the first row of the symmetric matrix $\bar{M}_{k}=\frac{1}{2}\left(M_{k}+M_{k}^{\top}\right)$. Doing this, we were able to find solutions with a simpler form, and felt that there was more hope in being able to write the solution vector $x_{k}$ in a Kronecker product calculation with the solution vector $x_{k-1}$. Noticing that all the entries in $M_{k}$ are integers, we found that it was possible to find a solution for $X_{k}$ in which all the entries in $X_{k}$ are integers, as far as $k=5$. It is not known whether this might be possible for even greater $k$. The $X_{k}$ constructed in the proof above have entries that are not integers, although they are rational.

Since $M_{k}$ is computed by Kronecker products it is natural to look for a solution vector of a form in which $x_{k}$ is expressed in terms of $x_{k-1}$ in some sort formula that also uses Kronecker products. The final breakthrough came in discovering the length 27 vector $(0,1,0) \otimes\left(a_{k}, a_{k}, 2,2, a_{k}, 2,1,1,1\right)$. This was found only after despairing of something simpler. We had hoped that if it were possible to find a Kronecker product form solution similar to $(22)$, then this would use a vector like the above, but of length only 3 or 9 . However, it was only when we tried something of length 27 that the final pieces fell in place. The final trick was to make the formula for obtaining $x_{k}$ from $x_{k-1}$ not be constant, but depending on $k$, as we have done with our $a_{k}$. We were lucky at the end that we could solve the recurrence relations for $t_{k 1}, t_{k 2}, t_{k 3}, t_{k 4}$ and prove $U_{k} x_{k} \geq 0$. It all looks so easy with hindsight!

4. Ongoing research It is as easy consequence of Theorem 1 that AW maximizes $E\left[\gamma^{T}\right]$ for all $\gamma \in(0,1)$. This follows from the fact that AW minimizes $\sum_{i=0}^{k} P(T>i)$ for all $k$.

We conjecture that AW is optimal in a rendezvous game played on three locations in which players may overlook one another with probability $\epsilon$, (that is, they can fail to meet even when they are in the same location). This is easily shown to be true for the game on two locations. The random strategy is optimal, with $E T=2 /(1-\epsilon)$. To analyse this game on three locations we redefine

$$
B_{1}=\left(\begin{array}{lll}
1 & 1 & \epsilon \\
\epsilon & 1 & 1 \\
1 & \epsilon & 1
\end{array}\right),
$$

where $0<\epsilon<1$. Now AW (with $p=1 / 3$ ) gives $E T=1+(3 / 2)(1+\epsilon) /(1-\epsilon)$. We can generalize all the ideas in the present paper, except that we have not been able to guess a construction for the matrix $X_{k}$. Fan (2009) [10] has observed that not only does AW appear to be optimal, but also that the optimal probability of 'staying' is the same for all $\epsilon$, i.e., $p=1 / 3$. However, for games on $K_{4}, K_{5}, \ldots$, the optimal value of $p$ is decreasing in $\epsilon$.

Of course one would very much like to have a direct proof that $\omega=5 / 2$, without needing to also find the $\omega_{k}$. Perhaps an idea for such a proof is hidden within the proof above. Or it may be found by further research on the open problem of rendezvous with overlooking, described above. 
While for many graphs it is possible to use the solution of a semidefinite programming problem to obtain a lower bound on the rendezvous value, it is not usually possible to recast the semidefinite programming problem as a linear program. A very important feature of our three locations problem is that it is so strongly captured within the algebra of a group of rotational symmetry, whose permutation matrices are the $P_{i}$. This continues to be true for rendezvous search on $C_{n}$, in which $n$ locations are arranged around a circle and players have a common notion of clockwise. The method in this paper is also effective in proving the optimality of a strategy for a simple but nontrivial rendezvous game that has been posed by John Howard. Two players are initially placed in two distinct locations. At each step a player can either stay where she is or move to the other location. When players are in the same location for the first time they do not know this and do not meet, but when they are in the same location for a second time then they meet. It is desired to minimized the time at which this occurs. We can show that the optimal strategy is this: that is each block of three steps a player should with equal probability do SSS, SMS, MMM, MSM, where 'M' means move and 'S' means stay, (see Weber [20]).

It will be interesting to explore whether our methods are helpful for rendezvous problems on other graphs. It is not hard to compute the optimal AW strategy for $n$ locations. See Anderson and Weber (1990) 8. For example, for $n=4$, the AW strategy achieves $E T=(1 / 12)(15+\sqrt{681}) \approx 3.4247$, using optimal probabilities of staying and touring of $p=(1 / 4)(3 \sqrt{681}-77) \approx 0.32198$ and $1-p \approx 0.6780$, respectively. As $n \rightarrow \infty$, an AW strategy achieves $E T \approx .8289 n$ with $p \approx 0.2475$. Interestingly, Fan (2009) [10] has shown that if the rendezvous game is played on four locations and locations are imagined to be placed around a circle, and players are provided with a common notion of clockwise, then there exists a 3-Markovian strategy that is better than AW. Recently, we have shown that even in the problem without common clockwise information the AW strategy is not optimal. The key idea is this: under the AW strategy on four locations a player is supposed to randomly tour his three 'non-home' locations or stay at home during each successive block of three steps. In fact, the expected rendezvous time can be reduced if players slightly modify AW so that that the tours that they make of their non-home locations are not just chosen at random amongst the six possible tours. By carefully making the choices of tours dependent one can find a strategy that has an expected rendezvous time that is about 0.000147 less than under AW. The better strategy is 12-Markovian (repeating over every four blocks of three steps). See Weber (2009) [19 for more details.

Another very interesting rendezvous search game is the one played on a line. The players start 2 units apart and can move 1 unit left or right at each step. In the asymmetric version (in which players I and II can adopt different strategies) the rendezvous value is known to be 3.25 (see Alpern and Gal, 1995 [5]). In the symmetric game work with semidefinite programming bounds has found that $4.1820 \leq \omega \leq 4.2574$ (see Han, et al. (2006) [11] and Alpern (2009) [4]). Alpern and Du have recently reported an improvement of the lower bound to 4.2326. At present, there are only numerical results for this problem. Han, et al. [11] have conjectured $w=4.25$. If this is correct then the difference in rendezvous values between the asymmetric and symmetric games is exactly 1 . This would make a surprising coincidence with what happens in the rendezvous search games on two locations and three locations, where the asymmetric rendezvous values for the games are 1 and 1.5 respectively (and achieved by the 'wait-for-mommy' strategy). These are also 1 less than the rendezvous values of 2 and 2.5 that pertain in the symmetric games (and are achieved by the AW strategy). However, we suspect that the rendezvous value of the symmetric game on the line is not 4.25. Indeed, an interesting line of research might be to try to prove that the rendezvous value of this game is neither irrational nor achieved by any $k$-Markovian strategy. Indeed it would be interesting to know of any symmetric rendezvous problem in which the optimal strategy is not Markovian. Another open question is whether an optimal strategy even exists.

A different conjecture about the games on three locations and on the line seems more likely. It is interesting that in the symmetric rendezvous search game on three locations it is of no help to the players to be provided with a common notion of clockwise. This has been called the Common Clockwise Conjecture. Indeed, Alpern and Gal (2006) [6] showed that if AW is optimal for three locations then this conjecture is true. Similarly, research on the symmetric rendezvous search game on the line suggests that it is no help to the players to be provided with a common notion of left and right. Of course rendezvous on the line can be viewed as rendezvous on $C_{n}$ as $n$ tends to infinity.

There are many interesting questions that remain open. A good source is Alpern [4]. Some unsolved 
problems are very simple. For instance, no one has yet proved the obvious conjecture that the rendezvous value for the symmetric rendezvous search game on $n$ locations is an increasing function of $n$.

Acknowledgments. I wish to warmly thank my Ph.D. student Junjie Fan. Without his enthusiasm for studing this problem I might not have had the courage to work on it once more. By pioneering the use of semidefinite programming as a method of addressing rendezvous search problems, Jimmy was the first in many years to obtain significantly improved lower bounds on the rendezvous value for three locations. The proof of Theorem 2.1 was first communicated to others in 2006, (see [18]). I am grateful to both Steve Alpern and John Howard for their creativity in posing such interesting problems and for the reading a preliminary draft.

\section{References}

[1] S. Alpern, Hide and seek games, Seminar at the Institut für Höhere Studien, Vienna, 26 July, 1976.

[2] _ _ The rendezvous search problem, SIAM J. Control Optim. 33 (1995), 673-683.

[3] _ Rendezvous search: A personal perspective, Oper. Res. 50 (2002), no. 5, 772-795.

[4] _ Rendezvous games (non-antagonistic search games), Wiley Encyclopedia of Operations Research and Management Sci. (James J. Cochran, ed.), Wiley, 2010, to appear.

[5] S. Alpern and S. Gal, The rendezvous problem on the line with distinguished players, SIAM J. Control Optim. 33 (1995), 1271-1277.

[6] _ Two conjectures on rendezvous in three locations, Tech. report, London School of Economics \& Political Science, December, 2006, http://www.cdam.lse.ac.uk/Reports/Abstracts/cdam-2006-21.html

[7] S. Alpern and M. Pikounis, The telephone coordination game., Game Theory Appl. 5 (2000), 1-10.

[8] E. J. Anderson and R. R. Weber, The rendezvous problem on discrete locations, J. Appl. Probab. 27 (1990), 839-851.

[9] V. P. Crawford and H. Haller, Learning how to cooperate: Optimal play in repeated coordination game, Econometrica 58 (1990), no. 3, 571-596.

[10] J. Fan, Symmetric rendezvous problem with overlooking, Ph.D. thesis, University of Cambridge, 2009.

[11] Q. Han, D. Du, J. C. Vera, and L. F. Zululaga, Improved bounds for the symmetric rendezvous problem on the line, Oper. Res. 56 (2006), no. 3, 772-782.

[12] D. Kafkewitz, Aisle miles, Why Don't Penguins' Feet Freeze?: And 114 Other Questions (M. O'Hare, ed.), Profile Books Ltd, 2006, pp. 202-205.

[13] D. R. Kowalski and A. Malinowski, How to meet in anonymous network, Theor. Comput. Sci. 399 (2008), no. 1-2, 141-156.

[14] F. Mosteller, Fifty challenging problems in probability with solutions, Dover, 1965.

[15] Rational Roots Theorem. http://en.wikipedia.org/wiki/Rational_root_theorem

[16] J. F. Sturm, Using sedumi 1.02, a Matlab toolbox for optimization over symmetric cones, Optim. Methods Softw. 11-12 (1999), 625-653.

[17] S. Venugopal, W. Chen, T. D. Todd, and K. Sivalingam, A rendezvous reservation protocol for energy constrained wireless infrastructure networks, Wireless Networks 13 (2007), no. 1, 93-105.

[18] R. R. Weber, The optimal strategy for symmetric rendezvous search on three locations, arXiv:0906.5447v1, November 2006.

[19] _ The Anderson-Weber strategy is not optimal for symmetric rendezvous search on four locations, arXiv:0912.0670v1, July 2009.

[20] _ Optimal strategy for Howard's rendezvous search game, under review. 